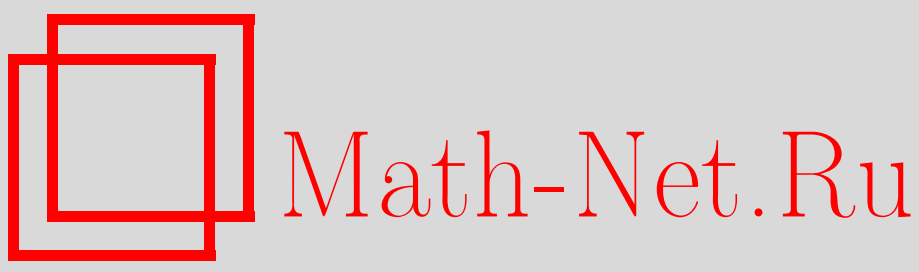

Е. А. Тевелев, Подалгебры и дискриминанты антикоммутативных алгебр, Изв. РАН. Сер. матем., 1999, том 63, выпуск 3, 169-184

DOI: https://doi.org/10.4213/im247

Использование Общероссийского математического портала Math-Net.Ru подразумевает, что вы прочитали и согласны с пользовательским соглашением http://www . mathnet.ru/rus/agreement

Параметры загрузки:

IP: 3.82 .47 .9

26 апреля 2023 г., $16: 44: 18$ 
УДК 512

\author{
Е. А. Тевелев
}

\title{
Подалгебры и дискриминанты антикоммутативных алгебр
}

\footnotetext{
В работе изучается конфигурация подалгебр в $n$-мерных $k$-местных антикоммутативных алгебрах общего положения, а также в "регулярных" антикоммутативных алгебрах.

Библиография: 11 наименований.
}

\section{Введение}

Пусть $V=\mathbb{C}^{n}$. Зафиксируем целое $k, 1<k<n-1$. Рассмотрим векторное пространство $\mathscr{A}_{n, k}=\Lambda^{k} V^{*} \otimes V \quad k$-линейных антикоммутативных отображений из $V$ в $V$. Мы будем отождествлять точки $\mathscr{A}_{n, k}$ с соответствуюшими алгебрами, т.е. $A \in \mathscr{A}_{n, k}$ всегда обозначает $V$ со структурой $k$-местной антикоммутативной алгебры.

1. Подалгебры в алгебрах общего положения. В случае $k=2$ подалгебры в алгебрах общего положения изучались в [11]. Следующая теорема обобщает эти результаты.

Теорема 1. Пусть $A \in \mathscr{A}_{n, k}$ - алгебра общего положения. Тогда:

1) всякое $m$-мерное подпространство является подалгеброй при $m<k$;

2) $A$ не содержит m-мерных подалгебр при $k+1<m<n$;

3) $k$-мерные подалгебры образуют гладкое неприводимое $(k-1)(n-k)$-мерное подмногообразие в грассманиане $\operatorname{Gr}(k, A)$;

4) число $(k+1)$-мерных подалгебр конечно и равно

$$
\begin{aligned}
& \sum_{\substack{n-k-1 \geqslant \mu_{1} \geqslant \cdots \geqslant \mu_{k+1} \geqslant 0 \\
n-k-1 \geqslant \lambda_{1} \geqslant \cdots \geqslant \lambda_{k+1} \geqslant 0 \\
\mu_{1} \leqslant \lambda_{1}, \ldots, \mu_{k+1} \leqslant \lambda_{k+1}}}(-1)^{|\mu|} \frac{\left(\lambda_{1}+k\right) !\left(\lambda_{2}+k-1\right) ! \ldots \lambda_{k+1} !}{\left(\mu_{1}+k\right) !\left(\mu_{2}+k-1\right) ! \ldots \mu_{k+1} !}(|\lambda|-|\mu|) ! \\
& \qquad\left|\frac{1}{\left(i-j+\lambda_{j}-\mu_{i}\right) !}\right|_{i, j=1, \ldots, k+1}^{2}, \\
& \text { 2де }|\lambda|=\lambda_{1}+\cdots+\lambda_{k+1},|\mu|=\mu_{1}+\cdots+\mu_{k+1} ; 1 / N !=0, \text { если } N<0 ; \\
& \text { 5) A содержит хотя бы одну }(k+1) \text {-мерную подалгебру; } \\
& \text { 6) при } k=n-2 \text { число }(k+1) \text {-мерных подалгебр равно }\left(2^{n}-(-1)^{n}\right) / 3 .
\end{aligned}
$$

Мы докажем теорему в $\S 1$. Приведем схему доказательства. Начнем со следующей общей ситуации.

Работа выполнена при финансовой поддержке фонда CRDF (грант RM1-206).

$$
\text { (C) Е.А. Тевелев } 1999
$$


Пусть $G$ - связная редуктивная группа, $T$ и $B$ - фиксированные максимальный тор и борелевская подгруппа, $T \subset B \subset G, B_{-}$- противоположная борелевская подгруппа, $B_{-} \subset P, P$ - параболическая подгрупп, $\mathbf{X}(T)$ - решетка характеров $T, \lambda \in \mathbf{X}(T)$ - доминантньй вес. Рассмотрим векторное расслоение $\mathscr{L}_{\lambda}=G \times_{P} U_{\lambda}$ над $G / P$, где $U_{\lambda}$ - неприводимьй $P$-модуль со старшим весом $\lambda$. По теореме Бореля-Вейля-Ботта (см. [7]) $G$-модуль $V_{\lambda}=H^{0}\left(G / P, \mathscr{L}_{\lambda}\right)$ является неприводимым модулем со старшим весом $\lambda$. Имеет место следуюшая простая лемма.

Лемма 1. Пусть $s \in V_{\lambda}-$ глобальное сечение общего положения. Тогда:

1) $п р и \operatorname{dim} U_{\lambda}>\operatorname{dim} G / P$ схема нулей $Z_{s}$ пуста;

2) $п р и \operatorname{dim} U_{\lambda} \leqslant \operatorname{dim} G / P$ либо $Z_{s}$ пуста, либо s пересекает нулевое сечение $\mathscr{L}_{\lambda}$ трансверсально и $Z_{s}$ - гладкое несмешанное подмногообразие коразмерности $\operatorname{dim} U_{\lambda}$;

3) $n р и \operatorname{dim} U_{\lambda}=\operatorname{dim} G / P$ геометрическое число точек в $Z_{s}$ равно стариему классу Чжсеня расслоения $\mathscr{L}_{\lambda}$.

Лемма будет доказана в $\S 1$. Покажем, как ее применить к доказательству теоремы. Как $\mathrm{GL}_{n}$-модуль $\mathscr{A}_{n, k}$ есть сумма двух неприводимых подмодулей:

$$
\mathscr{A}_{n, k}=\mathscr{A}_{n, k}^{0} \oplus \widetilde{\mathscr{A}}_{n, k}
$$

Здесь $\widetilde{\mathscr{A}}_{n, k}$ изоморфен $\Lambda^{k-1} V^{*}:$ каждой $(k-1)$-форме $\omega$ отвечает алгебра с умножением

$$
\left[v_{1}, \ldots, v_{k}\right]=\sum_{i=1}^{k}(-1)^{i-1} \omega\left(v_{1}, \ldots, \hat{v}_{i}, \ldots, v_{k}\right) v_{i} .
$$

Заметим, что всякое линейное подпространство такой алгебры является подалгеброй, поэтому структура подалгебр алгебры $A \in \mathscr{A}_{n, k}$ совпадает со структурой подалгебр алгебры $A^{0}$ (нулевой компоненты $A$ ), где $A \mapsto A^{0}-\mathrm{GL}_{n}$-эквивариантный проектор на первое слагаемое в (1). Алгебры из $\mathscr{A}_{n, k}^{0}$ будем называть алгебрами с нулевым следом, поскольку $A \in \mathscr{A}_{n, k}^{0}$ тогда и только тогда, когда $(k-1)$-форма $\operatorname{Tr}\left[v_{1}, \ldots, v_{k-1}, \cdot\right]$ равна нулю. Итак, для доказательства теоремы достаточно рассмотреть алгебры общего положения из $\mathscr{A}_{n, k}^{0}$.

Выберем в $V$ базис $\left\{e_{1}, \ldots, e_{n}\right\}$, отождествим $\mathrm{GL}_{n}$ с группой матрищ, выберем стандартный диагональный максимальный тор $T$, выберем в качестве $B$ и $B_{-}$подгруппы верхне- и нижнетреугольных матрищ. Зафиксируем $m \geqslant k$. Рассмотрим параболическую подгруппу матриц

$$
P=\left(\begin{array}{cc}
A & 0 \\
* & B
\end{array}\right)
$$

где $B-(m \times m)$-матрица, $A-((n-m) \times(n-m))$-матрица. Тогда $G / P$ - это грассманиан $\operatorname{Gr}(m, V)$. Рассмотрим на $G / P$ векторное расслоение $\mathscr{L}=\Lambda^{k} \mathscr{S}^{*} \otimes V / \mathscr{S}$, где $\mathscr{S}$ - тавтологическое расслоение, $V / \mathscr{S}$ - фактортавтологическое расслоение. Тогда мы находимся в ситуации леммы, поскольку, как легко видеть, $\mathscr{L}=\mathscr{L}_{\lambda}$, где $\lambda$ - старший вес $\mathscr{A}_{n, k}^{0}$. Таким образом, $\mathscr{A}_{n, k}^{0}=H^{0}\left(\operatorname{Gr}(m, V), \Lambda^{k} \mathscr{S}^{*} \otimes V / \mathscr{S}\right)$. 
Пусть $A \in \mathscr{A}_{n, k}^{0}, s_{A}$ - соответствуюшее глобальное сечение. Тогда $\left(Z_{s_{A}}\right)_{\text {red }}$ совпадает с многообразием $m$-мерных подалгебр $A$.

Вернемся к теореме. Пункт 1) очевиден, п. 2) следует из п. 1) леммы. Из п. 2) леммы следует, что если во всякой $k$-местной антикоммутативной алгебре $A$ есть $k$-мерная подалгебра, то многообразие $k$-мерных подалгебр алгебры общего положения - гладкое несмешанное $(k-1)(n-k)$-мерное подмногообразие на грассманиане $\operatorname{Gr}(k, A)$. Покажем, что произвольное $(k-1)$-мерное подпространство $U$ включается в $k$-мерную подалгебру.

Умножение в алгебре определяет линейное отображение из $V / U$ в $V / U$. Пусть $v+U$ - ненулевой собственный вектор. Тогда, очевидно, $\mathbb{C} v \oplus U-k$-мерная подалгебра. Для доказательства неприводимости многообразия $k$-мерных подалгебр мы используем комплекс Кошуля. Пп. 4) и 6) теоремы следуют из п. 3) леммы и явных вычислений в кольце Чжоу грассманиана $\operatorname{Gr}(k+1, V)$. Пункт 5$)$ теоремы потребует дополнительных вычислений.

Интересно отметить, что лемма 1 не может быть усилена таким образом, чтобы непустота и неприводимость схемы нулей в теореме 1 могла быть установлена a priori. Приведем пример. Рассмотрим расслоение $S^{2} \mathscr{S}^{*}$ на грассманиане $\operatorname{Gr}(k, 2 n)$. Тогда размерность слоя не превосходит размерности грассманиана при $k \leqslant(4 n-$ 1) $/ 3$, однако сечение обшего положения (т.е. невырожденная квадратичная форма в $\mathbb{C}^{2 n}$ ) имеет нуль (т.е. $k$-мерное изотропное подпространство) только при $k \leqslant n$. При этом для $k=n$ схема нулей - приводимое многообразие размерности $n(n-1) / 2$ с двумя неприводимыми компонентами, соответствующими двум семействам максимальных изотропных подпространств на четномерной квадрике.

Существенным недостатком теоремы является то, что она не позволяет выяснить структуру подалгебр никакой конкретной алгебры. Оставшаяся часть статьи частично исправляет это положение.

2. $D$-регулярные алгебры. Пусть $\mathscr{A}_{n, k}^{0^{*}}-\mathrm{GL}_{n}$-модуль, двойственный к $\mathscr{A}_{n, k}^{0}$, $S_{D}$ - замыкание орбиты старшего вектора, $S_{D} \subset \mathscr{A}_{n, k}^{0^{*}}$. Пусть $P S_{D} \subset P \mathscr{A}_{n, k}^{0^{*}}-$ его проективизация, $P \mathscr{D} \subset P \mathscr{A}_{n, k}^{0}-$ подмногообразие, проективно-двойственное (см. [2]) подмногообразию $P S_{D}, \mathscr{D} \subset \mathscr{A}_{n, k}^{0}-$ конус над ним. Тогда $\mathscr{D}$ называется $D$-дискриминантным подмногообразием. Алгебры $A \in \mathscr{D}$ назьваются $D$-сингулярными, алгебры $A \notin \mathscr{D}$ называются $D$-регулярными. Имеет место следующая теорема.

ТЕОРема 2. 1) Подмногообразие $\mathscr{D}$ является гиперповерхностью.

2) Пусть $A-D$-регулярная алгебра. Тогда $k$-мерные подалгебры $A$ образуют гладкое неприводимое $(k-1)(n-k)$-мерное подмногообразие на грассманиане $\operatorname{Gr}(k, A)$.

3) Пусть $k=n-2$. Тогда степень $\mathscr{D}$ равна

$$
\frac{\left(3 n^{2}-5 n\right) 2^{n}-4 n(-1)^{n}}{18}
$$

Таким образом, $D$-сингулярность алгебры $A$ определяется равенством нулю $\mathrm{SL}_{n}$-инвариантного полинома $D$, определяюшего $\mathscr{D}$. Этот полином называется 
$D$-дискриминантом. Теорема 2 будет доказана в $\oint 3$. Приведем схему доказательства. Тот факт, что $\mathscr{D}$ - гиперповерхность, немедленно следует из результатов [9]. Пункт 2) следует из соответствуюшего пункта теоремы 1 и несложных выкладок с дифференциалами. Пункт 3) требует некоторых пояснений. Нетрудно видеть, что $P S_{D}$ совпадает с многообразием неполных флагов $0 \subset V_{1} \subset V_{2} \subset \mathbb{C}^{n}$, где $\operatorname{dim} V_{1}=1$ и $\operatorname{dim} V_{2}=n-k$, в "плюккеровом" вложении. Поэтому трудно надеяться на то, что существует замкнутая формула для степени $\mathscr{D}$, поскольку нет хорошей формулы даже для степени многообразия, проективно-двойственного к грассманиану в плюккеровом вложении (см. [10], см. также формулу для многообразия, проективно-двойственного к многообразию полных флагов в [8]). Формулу (3) обобшает

Tеорема 2 $2^{\prime}$. Пусть $V^{*}$ - неприводимый $\mathrm{SL}_{n}$-модуль со стариим весом $(a-1) \varphi_{1}+\varphi_{2}$. Тогда многообразие $\mathscr{D} \subset P V$, проективно-двойственное $\kappa$ проективизачии орбить старшего вектора, является гиперповерхностью степени

$$
\frac{\left(n^{2}-n\right) a^{n+1}-\left(n^{2}+n\right) a^{n-1}-2 n(-1)^{n}}{(a+1)^{2}} .
$$

3. $E$-регулярные алгебры. При определении $E$-дискриминанта и $E$-регулярности мы ограничимся только случаем $(n-2)$-местных $n$-мерных антикоммутативных алгебр. Пусть $\mathscr{A}=\mathscr{A}_{n, n-2}^{0}$. Рассмотрим проекцию $\pi: \operatorname{Gr}(n-1, V) \times$ $P \mathscr{A} \rightarrow P \mathscr{A}$ на второе слагаемое и подмногообразие инцидентности $Z \subset$ $\operatorname{Gr}(n-1, V) \times P \mathscr{A}$, состояшее из пар $S \subset P A$, где $S$ - подалгебра в $A$. Пусть $\widetilde{\pi}=\left.\pi\right|_{Z}$. Тогда, как следует из теоремы $1, \widetilde{\pi}(Z)=\mathscr{A}$. Пусть $\widetilde{\mathscr{E}} \subset Z-$ множество критических точек $\widetilde{\pi}, P \mathscr{E}=\widetilde{\pi}(\widetilde{\mathscr{E}})$ - множество критических значений $\tilde{\pi}$, $\mathscr{E} \subset \mathscr{A}$ - конус над $P \mathscr{E}$. Тогда $\mathscr{E}$ называется $E$-дискриминантнылм подмногообразием. Алгебры $A \in \mathscr{E}$ называются $E$-сингулярнылми, алгебры $A \notin \mathscr{E}$ называются Е-регулярными. Имеет место следуюшая

ТЕОРема 3. 1) Подмногообразие Ё является неприводимой гиперповерхностью.

2) Пусть $A-$ Е-регулярная алгебра. Тогда в А ровно $\left(2^{n}-(-1)^{n}\right) / 3$ $(n-1)$-мерных подалгебр.

3) Отображение $\widetilde{\pi}: \widetilde{\mathscr{E}} \rightarrow$ РЁ бирационально.

Таким образом, $E$-сингулярность алгебры $A$ определяется равенством нулю $\mathrm{SL}_{n}$-инвариантного полинома $E$, определяющего $\mathscr{E}$. Этот полином называется E-дискриминантом. Пункт 3 ) можно переформулировать так: в $E$-сингулярной алгебре общего положения ровно одна "критическая" $(n-1)$-мерная подалгебра.

Теорема будет доказана в $\S 3$. Приведем схему доказательства. Пункт 1) следует из п. 3), неприводимости многообразия $\widetilde{\mathscr{E}}$ и вычисления его размерности. Пункт 2) следует из простых вычислений с дифференциалами. Таким образом, доказывать нужно будет п. 3).

4. Регулярные 4-мерные антикоммутативные алгебры. Назовем $(n-2)$-местную $n$-мерную антикоммутативную алгебру регулярной, если она $D$-регулярна и $E$-регулярна. В этом пункте мы рассматриваем 2-местные 4-мерные алгебры. Соответствуюшие алгебры обшего положения изучались в [11]. 
Приведем те свойства 4-мерных алгебр общего положения, которые выполняются уже во всех регулярных алгебрах.

ТЕОРема 4. Пусть $A-4$-мерная регулярная антикоммутативная алгебра. Тогда:

1) в А ровно пять 3-мерных подалгебр; эти подалгебры образуют конфигурацию из пяти гиперплоскостей общего положения; в частности, А обладает пентаәдральной нормальной формой, т.е. приводится преобразованием из $\mathrm{GL}_{4}$ $\kappa$ алгебре, пять подалгебр которой образуют пентаэдр Сильвестра $x_{1}=0$, $x_{2}=0, x_{3}=0, x_{4}=0, x_{1}+x_{2}+x_{3}+x_{4}=0$

2) в А нет одномерных и двумерных идеалов;

3) двумерные подалгебры А образуют поверхность дель Пецио степени 5 (раздутие $\mathbb{P}^{2}$ в четырех точках общего положения);

4) в А ровно 10 вееров, т.е. флагов $V_{1} \subset V_{2}$ из 1-мерного и 3-мерного подпространств таких, что всякое промежуточное подпространство $U$, $V_{1} \subset U \subset V_{3}$, является двумерной подалгеброй.

Доказательство будет дано в $\S 4$. Как показывают примеры, другие свойства алгебр общего положения, найденные в [11], вообще говоря, не выполнены в регулярных алгебрах. Например, можно привести примеры регулярных алгебр, в которых больше двух коммутативных подалгебр, в которых существуют трехмерные идеалы, для которых ассоциированная кубическая гиперповерхность не гладкая (и даже приводимая), и т.п.

Автор признателен своему научному руководителю Э. Б. Винбергу за полезные обсуждения и упрощения некоторых доказательств.

\section{§ 1. Подалгебры в алгебрах общего положения}

ДокАЗАТЕЛЬСтво ЛЕммы 1. Пункт 1) очевиден и доказан, например, в [11, лемма 1.1].

Докажем п. 2). Пусть $\operatorname{dim} U_{\lambda} \leqslant \operatorname{dim} G / P$ и всякое глобальное сечение имеет нуль. Нужно доказать, что глобальное сечение общего положения $s$ пересекает нулевое сечение $\mathscr{L}_{\lambda}$ трансверсально. Отсюда, в частности, будет следовать, что $Z_{s}$ - гладкое несмешанное подмногообразие коразмерности $\operatorname{dim} U_{\lambda}$. Для простоты опустим во всех обозначениях индекс $\lambda$. Рассмотрим произведение $G / P \times V$ и соответствие $Z \subset G / P \times V, Z=\left\{(x, s) \mid x \in\left(Z_{s}\right)_{\text {red }}\right\}$. Из однородности $G / P$ и инвариантности $Z$ следует, что $Z$ получается разнесением группой $G$ слоя $Z_{e}=\{s \in V \mid s(e P)=0\}$. Из неприводимости $U$ следует, что $\operatorname{dim} Z_{e}=\operatorname{dim} V-\operatorname{dim} U$, откуда $Z$ - гладкое неприводимое подмногообразие размерности $\operatorname{dim} V+\operatorname{dim} G / P-\operatorname{dim} U$.

Пусть $\pi: Z \rightarrow V$ - ограничение на $Z$ проекции $G / P \times V$ на второе слагаемое. По предположению $\pi$ сюръективно. По лемме Сарда для алгебраических многообразий (см. [4]) для точки $s \in V$ обшего положения и для любой точки $(x, s)$ из прообраза $\pi^{-1} s$ дифференциал $d \pi_{(x, s)}$ сюръективен. Покажем, что $s$ пересекает нулевое сечение трансверсально. Действительно, $\mathscr{L}=(G / P \times V) / Z$ как векторное расслоение над $G / P$. Нулевое сечение $\mathscr{L}$ отождествляется с $Z / Z$, 
а сечение $s-\mathrm{c}(G / P \times\{s\}) / Z$. Поэтому достаточно доказать трансверсальность $G / P \times\{s\}$ и $Z$. Но это равносильно сюръективности $d \pi_{(x, s)}$ для всех точек $(x, s) \in Z$. Теперь п. 3) леммы следует из стандартной теории пересечений (см. [5]): если схема нулей глобального сечения общего положения пуста, то $\mathscr{L}$ содержит тривиальное одномерное подрасслоение и его старший класс Чженя равен нулю, если не пуста, то используем п. 2).

ДоКАЗАТЕЛЬСТво тЕОРЕмЫ 1. Пункт 1) очевиден. Для доказательства остальных пунктов используем реализацию $\mathscr{A}_{n, k}^{0}$ как $H^{0}\left(\operatorname{Gr}(m, V), \Lambda^{k} \mathscr{S}^{*} \otimes V / \mathscr{S}\right)$. Пп. 2) и 3 ) непосредственно следует из леммы 1 , нужно только доказать неприводимость многообразия $k$-мерных подалгебр. Пусть сечение $s$ расслоения $\mathscr{L}=\Lambda^{k} \mathscr{S}^{*} \otimes V / \mathscr{S}$ над $\operatorname{Gr}(k, V)$, соответствуюшее алгебре $A$, пересекает нулевое сечение трансверсально. Тогда комплекс Кошуля

$$
0 \rightarrow \Lambda^{n-k} \mathscr{L}^{*} \stackrel{s}{\rightarrow} \cdots \stackrel{s}{\rightarrow} \Lambda^{2} \mathscr{L}^{*} \stackrel{s}{\rightarrow} \mathscr{L}^{*} \stackrel{s}{\rightarrow} \mathscr{O} \rightarrow \mathscr{O}_{Z(s)} \rightarrow 0
$$

точен (см. [5]).

Заметим, что $\Lambda^{p} \mathscr{L}^{*}$ изоморфно расслоению $S^{p} \Lambda^{k} \mathscr{S} \otimes \Lambda^{p}(V / \mathscr{S})^{*}$. Это однородное расслоение над $G / P$ вида $\mathscr{L}_{\mu}$ (см. введение), где $\mu=-\varepsilon_{1}-\cdots-\varepsilon_{p}+$ $\varepsilon_{n-k+1}+\cdots+\varepsilon_{n} ; \varepsilon_{i}-$ веса диагонального тора в тавтологическом представлении. Заметим, что для любого $p, \quad 1 \leqslant p \leqslant n-k$, вес $\mu+\rho$ (здесь $\rho$ - полусумма положительных корней) сингулярен (лежит на стенке камеры Вейля), поэтому по теореме Бореля-Вейля-Ботта $H^{*}\left(\operatorname{Gr}(k, V), \Lambda^{p} \mathscr{L}^{*}\right)=0$ при $1 \leqslant p \leqslant n-k$. Отсюда $H^{0}\left(Z(s), \mathscr{O}_{Z(s)}\right)=H^{0}(\operatorname{Gr}(k, V), \mathscr{O})=\mathbb{C}$, что и требовалось доказать.

Отложим доказательство п. 5) до конща этого параграфа, а пока займемся пп. 4) и 6), т.е. вычислением старшего класса Чженя расслоения $\Lambda^{k} \mathscr{S}^{*} \otimes V / \mathscr{S}$ на грассманиане $\operatorname{Gr}(k+1, V)$.

Используем стандартные обозначения, факты и формулы из исчисления Шуберта (ср. [5]). Буквы $\lambda$ и $\mu$ всегда обозначают диаграммы Юнга, содержашиеся в прямоугольнике из $k+1$ строк и $n-k-1$ столбцов. Известно, что такие диаграммы параметризуют базис кольца Чжоу грассманиана $\operatorname{Gr}(k+1, V)$; цикл, соответствующий диаграмме $\lambda$, обозначается $\sigma_{\lambda}, \sigma_{\lambda} \in A^{|\lambda|}(\operatorname{Gr}(k+1, V))$ (мы градуируем кольцо Чжоу по коразмерности, $|\lambda|=\lambda_{1}+\cdots+\lambda_{k+1}$, где $\lambda_{i}$ - длина $i$-й строки диаграммы $\lambda$ ). Нам понадобится полньй класс Чженя расслоений $\mathscr{S}$ и $V / \mathscr{S}$ :

$c(\mathscr{S})=1-\sigma_{1}+\sigma_{1,1}-\cdots+(-1)^{k+1} \sigma_{1, \ldots, 1}, \quad c(V / \mathscr{S})=1+\sigma_{1}+\sigma_{2}+\cdots+\sigma_{n-k-1}$.

Начинаем с вычисления полного класса Чженя расслоения $\mathscr{S} \otimes V / \mathscr{S}$. Стандартная формула для полного класса Чженя тензорного произведения двух расслоений дает

$$
c(\mathscr{S} \otimes V / \mathscr{S})=\sum_{\mu \subset \lambda} d_{\lambda \mu} \Delta_{\widetilde{\mu}}(c(\mathscr{S})) \Delta_{\lambda^{\prime}}(c(V / \mathscr{S})),
$$

где

$$
\begin{aligned}
d_{\lambda \mu} & =\left|\left(\begin{array}{c}
\lambda_{i}+k+1-i \\
\mu_{j}+k+1-j
\end{array}\right)\right|_{1 \leqslant i, j \leqslant k+1}, \quad \Delta_{\lambda}(c)=\left|c_{\lambda_{i}+j-i}\right|, \\
\lambda^{\prime} & =\left(n-k-1-\lambda_{k+1}, n-k-1-\lambda_{k}, \ldots, n-k-1-\lambda_{1}\right),
\end{aligned}
$$


$\widetilde{\mu}$ - диаграмма, которая получается из $\mu$ транспонированием. Далее мы используем то, что $\Delta_{\widetilde{\mu}}(c(\mathscr{S}))=\Delta_{\mu}(s(\mathscr{S})) ; s(E)-$ класс Сегре расслоения $E$. Еще один факт состоит в том, что $\Delta_{\lambda}(c(V / \mathscr{S}))=\sigma_{\lambda}, \Delta_{\lambda}(s(\mathscr{S}))=(-1)^{|\lambda|} \sigma_{\lambda}$ (поскольку $\left.s(\mathscr{S})=1-\sigma_{1}+\sigma_{2}+\cdots+(-1)^{n-k-1} \sigma_{n-k-1}\right)$. Поэтому (4) переписывается в виде

$$
c(\mathscr{S} \otimes V / \mathscr{S})=\sum_{\mu \subset \lambda} d_{\lambda \mu}(-1)^{|\mu|} \sigma_{\mu} \sigma_{\lambda^{\prime}}
$$

Теперь начнем вычислять старший класс Чженя расслоения $\mathscr{L}=\Lambda^{k} \mathscr{S}^{*} \otimes V / \mathscr{S}$. Используем то, что $\mathscr{L}=\Lambda^{k+1} \mathscr{S}^{*} \otimes(\mathscr{S} \otimes V / \mathscr{S})$. Полный класс Чженя первого сомножителя равен $c\left(\Lambda^{k+1} \mathscr{S}^{*}\right)=1+\sigma_{1}$, а правого сомножителя дается формулой (5). Поэтому старший класс Чженя расслоения $\mathscr{L}$ есть

$$
c_{\text {top }}(\mathscr{L})=\sum_{\mu \subset \lambda} d_{\lambda \mu}(-1)^{|\mu|} \sigma_{\mu} \sigma_{\lambda^{\prime}} \sigma_{1}^{|\lambda|-|\mu|}
$$

Последний результат из исчисления Шуберта, который мы используем, это точная формула для степени произведения двух циклов. В нашем случае она дает

$$
\sigma_{\mu} \sigma_{\lambda^{\prime}} \sigma_{1}^{|\lambda|-|\mu|}=\operatorname{deg}\left(\sigma_{\mu} \sigma_{\lambda^{\prime}}\right)=(|\lambda|-|\mu|) !\left|\frac{1}{\left(i-j+\lambda_{j}-\mu_{i}\right) !}\right|_{i, j=1, \ldots, n},
$$

здесь $1 / N !=0$, если $N<0$. Формула из п. 4) теоремы 1 получается из формул (6) и $(7)$, после небольшой модификации определителя в формуле для $d_{\lambda \mu}$.

Осталось проверить формулу из п. 6) теоремы 1 . Пусть $k=n-2$. Тогда формула из п. 4) перепишется в виде

$$
\begin{aligned}
\sum_{0 \leqslant i \leqslant j \leqslant k+1}(-1) \frac{(1+k) !(1+(k-1)) ! \ldots(1+(k-j+1)) !(k-j) ! \ldots 0 !}{(1+k) !(1+(k-1)) ! \ldots(1+(k-i+1)) !(k-i) ! \ldots 0 !} \\
\quad \times(j-i) ! \operatorname{det}^{2} A
\end{aligned}
$$

где

$$
A=\left(\begin{array}{ccc}
X & 0 & 0 \\
* & Y & 0 \\
* & * & Z
\end{array}\right) .
$$

Здесь $X-(i \times i)$-матрица, $Y-((j-i) \times(j-i))$-матрица, $Z-((k+1-j) \times$ $(k+1-j))$-матрица. Матрицы $X$ и $Z$ нижнетреугольные с единицами на диагонали, а матрища $Y$ имеет вид

$$
Y=\left(\begin{array}{cccccc}
1 & 1 & 0 & 0 & \ldots & 0 \\
1 / 2 ! & 1 & 1 & 0 & \ldots & 0 \\
1 / 3 ! & 1 / 2 ! & 1 & 1 & \ldots & 0 \\
1 / 4 ! & 1 / 3 ! & 1 / 2 ! & 1 & \ldots & 0 \\
\ldots \ldots \ldots \ldots \ldots \ldots \ldots \ldots \ldots \ldots \ldots \ldots \ldots \ldots \ldots \ldots \ldots \ldots \ldots \ldots \ldots & \ldots \ldots \ldots
\end{array}\right)
$$


Нетрудно проверить, что $\operatorname{det} Y=1 /(j-i)$ !, откуда формула (8) переписьвается в виде

$$
\begin{gathered}
\sum_{i \leqslant j}(-1)^{i} \frac{(1+(k-i)) ! \ldots(1+(k-j+1)) !}{(k-i) ! \ldots(k-j+1) !} /(j-i) !=\sum_{i \leqslant j}(-1)^{i}\left(\begin{array}{c}
k+1-i \\
j-i
\end{array}\right) \\
\quad=\sum_{i}(-1)^{i} 2^{k+1-i}=\frac{2^{k+2}-(-1)^{k+2}}{3}=\frac{2^{n}-(-1)^{n}}{3},
\end{gathered}
$$

что и требовалось доказать.

Осталось доказать п. 5) теоремы 1. Нужно доказать, что всякая алгебра $A \in \mathscr{A}_{n, k}$ имеет $(k+1)$-мерную подалгебру. Зафиксируем в $V$ базис $\left\{e_{1}, \ldots, e_{n}\right\}$ и рассмотрим подпространство $U=\left\langle e_{n-k}, \ldots, e_{n}\right\rangle$. Пусть $M \subset \mathscr{A}_{n, k}-$ линейное подпространство всех алгебр, для которых $U$ является $(k+1)$-мерной подалгеброй. Мы хотим доказать, что $\mathscr{A}_{n, k}=\mathrm{GL}_{n} \cdot M$. Для этого достаточно проверить, что дифференциал канонического морфизма $\phi: \mathrm{GL}_{n} \times M \rightarrow \mathscr{A}_{n, k}$ сюръективен в некоторой точке вида $(e, A)$. Возьмем алгебру $A \in M$, в которой $\left[e_{n-k}, \ldots, \hat{e}_{i}, \ldots, e_{n}\right]=e_{i}$ при всех $n-k \leqslant i \leqslant n$, а все остальные произведения равны нулю. Покажем, что $d \phi$ сюръективен в точке $(e, A)$. Рассмотрим отображение $\pi: \mathscr{A}_{n, k} \rightarrow \mathscr{A}_{n, k} / M$. Достаточно проверить, что

$$
\pi \circ d \phi_{(e, A)}\left(\mathfrak{g l}_{n}, 0\right)=\mathscr{A}_{n, k} / M \simeq \Lambda^{k} U^{*} \otimes V / U
$$

Но это очевидно, поскольку при $n-k \leqslant i \leqslant n, 1 \leqslant j \leqslant n-k-1$ умножениев алгебре $d \phi_{(e, A)}\left(E_{j i}, 0\right)\left(E_{j i}-\right.$ матричная единица) имеет вид $\left[e_{n-k}, \ldots, \hat{e}_{i}, \ldots, e_{n}\right]=e_{j}$, все остальные произведения равны нулю. Теорема доказана.

\section{§ 2. D-регулярные алгебры}

ДокАЗАТЕЛЬСТво теорем 2 и $2^{\prime}$. В работе [9] найдены все неприводимые представления полупростых групп, для которых многообразие, двойственное к проективизации орбиты старшего вектора, не является гиперповерхностью. Поскольку в этом списке нет $\mathscr{A}_{n, k}^{0^{*}}$ (как $\mathrm{SL}_{n}$-модуля), то $\mathscr{D}$ является гиперповерхностью. Таким образом, дискриминант $D$ корректно определен.

Докажем п. 2). Пусть $A-D$-регулярная алгебра. Мы используем соображения из доказательства п. 2) леммы 1 и п. 3) теоремы 1. В соответствии с этими вычислениями нам достаточно проверить, что если $U-k$-мерная подалгебра $A$, то отображение

$$
\begin{gathered}
\psi: \mathfrak{g l}_{n} \rightarrow \Lambda^{k} U \otimes A / U \\
\psi(g)\left(v_{1} \wedge \cdots \wedge v_{k}\right)=g\left[v_{1}, \ldots, v_{k}\right]-\left[g v_{1}, \ldots, v_{k}\right]-\cdots-\left[v_{1}, v_{2}, \ldots, g v_{k}\right]+U
\end{gathered}
$$

сюръективно. Пусть это не так. Тогда существует гиперплоскость $H \supset U$ такая, что образ $\psi$ лежит в $\Lambda^{k} U \otimes H / U$. Рассмотрим ненулевую алгебру $\widetilde{A}$ из $S_{D} \subset \mathscr{A}_{n, k}^{0^{*}}$ такую, что

$$
\left[U^{\perp}, V^{*}, \ldots, V^{*}\right]=0, \quad\left[V^{*}, \ldots, V^{*}\right] \subset H^{\perp}
$$


где $U^{\perp}, H^{\perp}$ - аннуляторы $U$ и $H$ в $\mathscr{A}_{n, k}^{0^{*}}$ (такая алгебра единственна с точностью до скаляра). Тогда $\widetilde{A}$ аннулирует $\left[\mathfrak{g l}_{n}, A\right]$, или, что эквивалентно, $A$ аннулирует $\left[\mathfrak{g l}_{n}, \widetilde{A}\right]$, т.е. касательное пространство к $S_{D}$ в точке $\widetilde{A}$. Но это и означает, что $A$ лежит в $\mathscr{D}$, т.е. является $D$-сингулярной алгеброй.

Далее мы предполагаем, что $k=n-2$. Поскольку п. 3) теоремы 2 следует из теоремы $2^{\prime}$ (при $a=2$ ), достаточно доказать теорему $2^{\prime}$. Нам нужно вычислить степень $\mathscr{D}$. Мы используем формулу Клеймана (см. [2]) для степени двойственного многообразия: если $Z$ - гладкое проективное $l$-мерное многообразие в $\mathbb{P}^{n-1}$ и $\mathscr{L}=\left.\mathscr{O}_{\mathbb{P}^{n-1}}(1)\right|_{Z}$, то

$$
\operatorname{deg}(\check{Z})=\sum_{i=0}^{l}(i+1) \int_{Z} c_{l-i}\left(\Omega_{Z}^{1}\right) c_{1}(\mathscr{L})^{i},
$$

где $\check{Z}$ - проективно-двойственное многообразие.

В нашем случае $Z=G / P$, где $G=\mathrm{GL}_{n}, P \subset G$ - параболическая подгруппа матриц

$$
\left(\begin{array}{ccccc}
* & 0 & 0 & \ldots & 0 \\
* & * & 0 & \ldots & 0 \\
* & * & * & \ldots & * \\
\ldots \ldots & \ldots & \ldots & . \\
* & * & * & \ldots & *
\end{array}\right)
$$

Пусть $T \subset G$ - диагональный тор, $B$ - борелевская подгруппа нижнетреугольных матриц, $x_{1}, \ldots, x_{n}$ - веса тавтологического представления, $X(T)$ - решетка характеров $T, S$ - симметрическая алгебра $X(T)$ (над $\mathbb{Q}$ ), $W \simeq S_{n}$ - группа Вейля $G$, $W_{P} \simeq S_{n-2}$ - группа Вейля $P$. Хорошо известно (см. [1]), что отображение $c: X(T) \rightarrow \operatorname{Pic}(G / B)$, сопоставляющее весу $\lambda$ первый класс Чженя обратимого пучка $\mathscr{L}_{\lambda}$ (см. введение), продолжается до сюръективного гомоморфизма $c: S \rightarrow A^{*}(G / B)$ в (рациональное) кольцо Чжоу, причем его ядро совпадает с $S_{+}^{W} S$. Проекция $\alpha: G / B \rightarrow G / P$ индуцирует вложение $\alpha^{*}: A^{*}(G / B) \rightarrow A^{*}(G / B)$, причем образ совпадает с подалгеброй $W_{P}$-инвариантов. Таким образом, $A^{*}(G / P)=S^{W_{P}} / S_{+}^{W} S^{W_{P}}$. Будем обозначать гомоморфизм $S^{W_{P}} \rightarrow A^{*}(G / P)$ тоже буквой $c$.

Для применения формулы Клеймана нам нужен класс $c_{1}(\mathscr{L})$ (но он по построению равен $\left.c\left(a x_{1}+x_{2}\right)\right)$ и полный класс Чженя расслоения $\Omega_{Z}^{1}$, который равен

$$
c\left(\Omega_{Z}^{1}\right)=c\left(\left(1-x_{1}+x_{2}\right) \prod_{i=3, \ldots, n}\left(1-x_{1}+x_{i}\right) \prod_{i=3, \ldots, n}\left(1-x_{2}+x_{i}\right)\right)
$$

(как показывают стандартные аргументы, используюшие фильтрацию $\Omega_{Z}^{1}$; см. также [8]).

Пусть $\alpha_{1}, \ldots, \alpha_{n-2}$ - элементарные симметрические многочлены от набора переменных $x_{3}, \ldots, x_{n}$. Тогда $S^{W_{P}}=\mathbb{Q}\left[x_{1}, x_{2}, \alpha_{1}, \ldots, \alpha_{n-2}\right]$, а идеал $S_{+}^{W} S^{W_{P}}$ порождается элементами

$$
\begin{gathered}
x_{1}+x_{2}+\alpha_{1}, \quad x_{1} x_{2}+x_{1} \alpha_{1}+x_{2} \alpha_{1}+\alpha_{2}, \\
x_{1} x_{2} \alpha_{1}+x_{1} \alpha_{2}+x_{2} \alpha_{2}+\alpha_{3}, \ldots, x_{1} x_{2} \alpha_{n-4}+x_{1} \alpha_{n-3}+x_{2} \alpha_{n-3}+\alpha_{n-2}, \\
x_{1} x_{2} \alpha_{n-3}+x_{1} \alpha_{n-2}+x_{2} \alpha_{n-2}, \quad x_{1} x_{2} \alpha_{n-2} .
\end{gathered}
$$


Отсюда $\alpha_{i}=(-1)^{i}\left(x_{1}^{i}+x_{1}^{i-1} x_{2}+\cdots+x_{2}^{i}\right) \bmod S_{+}^{W} S^{W_{P}}$ и $A^{*}(G / P)$ изоморфно факторкольцу $\mathbb{Q}\left[x_{1}, x_{2}\right] /\left\langle f_{1}, f_{2}\right\rangle$, где $f_{1}=x_{1}^{n-1}+x_{1}^{n-2} x_{2}+\cdots+x_{2}^{n-1}, f_{2}=x_{1}^{n}$. Заметим, что $f_{1}, f_{2}$ - базис Гребнера идеала $\left\langle f_{1}, f_{2}\right\rangle$ относительно порядка $x_{2}>x_{1}$ (см. [6]); поэтому элементы $X^{i} Y^{j}$, где $X=x_{1} \bmod \left\langle f_{1}, f_{2}\right\rangle, Y=x_{2} \bmod \left\langle f_{1}, f_{2}\right\rangle$, $i=1, \ldots, n-1, j=1, \ldots, n-2$, образуют базис факторалгебры.

Для того чтобы вычислить степень дискриминанта по формуле Клеймана, нужно еще вычислить $\int_{Z} c\left(X^{n-1} Y^{n-2}\right)$. Пусть $\widetilde{w}_{0}$ - самьй длинный элемент группы $W, w_{0}$ - самый короткий элемент в $\widetilde{w}_{0} W_{P}$ с приведенным разложением

$$
\begin{aligned}
w_{0} & =\left(\begin{array}{cccccc}
1 & 2 & 3 & 4 & \ldots & n \\
n & n-1 & 1 & 2 & \ldots & n-2
\end{array}\right) \\
& =(n-1, n)(n-2, n-1) \ldots(12) \cdot(n-1, n)(n-2, n-1) \ldots(23) .
\end{aligned}
$$

Пусть $\quad A_{w_{0}}=A_{(n-1, n)} A_{(n-2, n-1)} \ldots A_{(12)} A_{(n-1, n)} A_{(n-2, n-1)} \ldots A_{(23)} \quad-$ соответствуюший эндоморфизм степени $-(2 n-3)$ в $S$, где $A_{(i j)}=$ $\left(\mathrm{id}-s_{(i j)}\right) /\left(x_{i}-x_{j}\right)$, а $s_{(i j)}$ - отражение, меняющее местами $x_{i}$ и $x_{j}$. Тогда $\int_{Z} c\left(X^{n-1} Y^{n-2}\right)=A_{w_{0}}\left(x_{1}^{n-1} x_{2}^{n-2}\right)$ (см. [1]). Однако нетрудно видеть, что

$$
\begin{aligned}
A_{w_{0}}\left(x_{1}^{n-1} x_{2}^{n-2}\right) & =A_{\rho_{1}} A_{\rho_{2}}\left(x_{1}^{n-1} x_{2}^{n-2}\right)=A_{\rho_{1}}\left(x_{1}^{n-1} A_{\rho_{2}}\left(x_{2}^{n-2}\right)\right) \\
& =A_{\rho_{1}}\left(x_{1}\right) A_{\rho_{2}}\left(x_{2}\right)
\end{aligned}
$$

где $\rho_{k}=(n-1, n)(n-2, n-1) \ldots(k, k+1)$. Оба эти сомножителя равны 1 , поскольку они равны $\int_{\mathbb{P}^{n-1}} c_{1}(\mathscr{O}(1))^{n-1}$ и $\int_{\mathbb{P}^{n-2}} c_{1}(\mathscr{O}(1))^{n-2}$ соответственно, а эти интегралы, очевидно, равны 1 . Окончательно получаем, что $\int_{Z} c\left(X^{n-1} Y^{n-2}\right)=1$.

Нам осталось вычислить в кольце $\mathbb{Q}[X, Y]$ (с базисом $X^{i} Y^{j}, i=1, \ldots, n-1$, $j=1, \ldots, n-2$, соотношениями $X^{n-1}+X^{n-2} Y+\cdots+Y^{n-1}=0, \quad Y^{n}=0$ и следуюшим из них соотношением $X^{n}=0$ ) многочлен

$$
\sum_{i=0}^{2 n-3}(i+1) c_{2 n-3-i}(a X+Y)^{i}
$$

где $c_{k}-k$-я однородная компонента многочлена

$$
(1-X+Y) \prod_{i=3}^{n}\left(1-X+x_{i}\right) \prod_{i=3}^{n}\left(1-Y+x_{i}\right)
$$

в котором $i$-ю симметрическую функцию от переменных $x_{3}, \ldots, x_{n}$ нужно заменить на $(-1)^{i}\left(X^{i}+X^{i-1} Y+\cdots+Y^{i}\right)$. Результатом вычисления будет $\operatorname{deg}(\mathscr{D}) X^{n-1} Y^{n-2}$.

Заметим, что многочлен (10) равен

$$
\left.F^{\prime}(T)\right|_{T=2 X+Y}=\left.\left(F_{1} F_{2} F_{3} F_{4}\right)^{\prime}(T)\right|_{T=2 X+Y},
$$


где

$$
\begin{gathered}
F_{1}=T, \quad F_{2}=T-X+Y, \\
F_{3}=\prod_{i=3}^{n}\left(T-X+x_{i}\right)=\sum_{i=0}^{n-2}(T-X)^{n-2-i}(-1)^{i}\left(X^{i}+\cdots+Y^{i}\right), \\
F_{4}=\prod_{i=3}^{n}\left(T-Y+x_{i}\right)=\sum_{i=0}^{n-2}(T-Y)^{n-2-i}(-1)^{i}\left(X^{i}+\cdots+Y^{i}\right) .
\end{gathered}
$$

Далее,

$$
\begin{aligned}
F_{3}(T)= & \left(\sum_{i=0}^{n-2}(T-X)^{n-2-i}(-1)^{i} \frac{X^{i+1}-Y^{i+1}}{X-Y}\right) \\
= & \frac{X(T-X)^{n-2}}{X-Y}\left(\sum_{i=0}^{n-2}(T-X)^{-i}(-1)^{i} X^{i}\right) \\
& -\frac{Y(T-X)^{n-2}}{X-Y}\left(\sum_{i=0}^{n-2}(T-X)^{-i}(-1)^{i} Y^{i}\right) \\
= & \frac{X\left((T-X)^{n-1}-(-X)^{n-1}\right)}{T(X-Y)}-\frac{Y\left((T-X)^{n-1}-(-Y)^{n-1}\right)}{(T-X+Y)(X-Y)}
\end{aligned}
$$

Аналогично,

$$
\begin{aligned}
F_{4}(T)= & \left(\sum_{i=0}^{n-2}(T-Y)^{n-2-i}(-1)^{i} \frac{X^{i+1}-Y^{i+1}}{X-Y}\right) \\
= & \frac{X(T-Y)^{n-2}}{X-Y}\left(\sum_{i=0}^{n-2}(T-Y)^{-i}(-1)^{i} X^{i}\right) \\
& -\frac{Y(T-Y)^{n-2}}{X-Y}\left(\sum_{i=0}^{n-2}(T-Y)^{-i}(-1)^{i} Y^{i}\right) \\
= & \frac{X\left((T-Y)^{n-1}-(-X)^{n-1}\right)}{(T+X-Y)(X-Y)}-\frac{Y\left((T-Y)^{n-1}-(-Y)^{n-1}\right)}{T(X-Y)} .
\end{aligned}
$$

Отсюда после некоторых вычислений получаем

$$
\begin{aligned}
F^{\prime}(T)= & \frac{n(X-Y)(T-X)^{n-1}+(-X)^{n}-(-Y)^{n}}{X-Y} \\
& \times \frac{(X-Y)(T-Y)^{n}+T(-X)^{n}-(T+X-Y)(-Y)^{n}}{T(T+X-Y)(X-Y)} \\
& +\frac{(X-Y)(T-X)^{n}+(T-X+Y)(-X)^{n}-T(-Y)^{n}}{T(X-Y)} \\
& \times \frac{(n(T+X-Y)-(T-Y))(T-Y)^{n-1}+(-X)^{n}}{(T+X-Y)^{2}} \\
& -\frac{(X-Y)(T-X)^{n}+(T-X+Y)(-X)^{n}-T(-Y)^{n}}{T(X-Y)} \\
& \times \frac{(X-Y)(T-Y)^{n}+T(-X)^{n}-(T+X-Y)(-Y)^{n}}{T(T+X-Y)(X-Y)} .
\end{aligned}
$$


Используем элементарные формулы

$$
\begin{aligned}
\frac{\alpha^{n}(\gamma-\beta)+\beta^{n}(\alpha-\gamma)+\gamma^{n}(\beta-\alpha)}{(\alpha-\beta)(\beta-\gamma)(\gamma-\alpha)} & =\sum_{i+j+k=n-2} \alpha^{i} \beta^{j} \gamma^{k} \\
\frac{\alpha^{n}(\gamma-\beta)+\beta^{n}(\alpha-\gamma)+\gamma^{n}(\beta-\alpha)}{(\alpha-\beta)(\gamma-\beta)} & =\sum_{i=0}^{n-2} \beta^{i}\left(\alpha^{n-1-i}-\gamma^{n-1-i}\right), \\
\frac{(n(\alpha-\beta)-\alpha) \alpha^{n-1}+\beta^{n}}{(\alpha-\beta)^{2}} & =\sum_{i=0}^{n-2}(n-1-i) \alpha^{n-2-i} \beta^{i} .
\end{aligned}
$$

Получим

$$
\begin{aligned}
F^{\prime}(T)= & \left(n(T-X)^{n-1}+(-1)^{n} \sum_{i=0}^{n-1} X^{n-1-i} Y^{i}\right) \\
& \times\left(\sum_{i+j+k=n-2}(T-Y)^{i}(-X)^{j}(-Y)^{k}\right) \\
& +\left(\sum_{i=0}^{n-2}(-X)^{i}\left((T-X)^{n-1-i}-(-Y)^{n-1-i}\right)\right) \\
& \times\left(\sum_{i=0}^{n-2}(n-1-i)(T-Y)^{n-2-i}(-X)^{i}\right) \\
& -\left(\sum_{i=0}^{n-2}(-X)^{i}\left((T-X)^{n-1-i}-(-Y)^{n-1-i}\right)\right) \\
& \times\left(\sum_{i+j+k=n-2}(T-Y)^{i}(-X)^{j}(-Y)^{k}\right) .
\end{aligned}
$$

Положим $T=a X+b Y$ и подставим в эту формулу. Получим

$$
\begin{aligned}
& \left(\sum_{i=0}^{n-1}\left(1+(-1)^{n} n(a-1)^{n-1-i} b^{i}\left(\begin{array}{c}
n-1 \\
i
\end{array}\right)\right) X^{n-1-i} Y^{i}\right) \\
& \quad \times\left(\sum_{i=0}^{n-2}\left[\begin{array}{c}
n-2 \\
i
\end{array}\right]_{a, b-1} X^{i} Y^{n-2-i}\right) \\
& \quad-\left(\sum_{i=0}^{n-1}\left(\left[\begin{array}{c}
n-1 \\
i
\end{array}\right]_{a-1, b}-\left[\begin{array}{c}
n-2 \\
i-1
\end{array}\right]_{a-1, b}-1\right) X^{n-1-i} Y^{i}\right) \\
& \quad \times\left(\sum_{i=0}^{n-2}\left(\left\{\begin{array}{c}
n-2 \\
i
\end{array}\right\}_{a, b-1}-\left\{\begin{array}{c}
n-3 \\
i
\end{array}\right\}_{a, b-1}\right) X^{n-2-i} Y^{i}\right) \\
& \quad+\left(\sum_{i=0}^{n-1}\left(\left[\begin{array}{c}
n-1 \\
i
\end{array}\right]_{a-1, b}-\left[\begin{array}{c}
n-2 \\
i-1
\end{array}\right]_{a-1, b}-1\right) X^{i} Y^{n-1-i}\right) \\
& \quad \times\left(\sum_{i=0}^{n-2}\left[\begin{array}{c}
n-2 \\
i
\end{array}\right]_{a, b-1} X^{i} Y^{n-2-i}\right),
\end{aligned}
$$


где

$$
\begin{aligned}
& {\left[\begin{array}{l}
n \\
k
\end{array}\right]_{x y}=\sum_{p=0}^{k} \sum_{q=0}^{n-k}(-1)^{p+q} x^{p} y^{q}\left(\begin{array}{c}
p+q \\
p
\end{array}\right),} \\
& \left\{\begin{array}{l}
n \\
k
\end{array}\right\}_{x y}=\sum_{p=0}^{k} \sum_{q=0}^{n-k}(p+q+1)(-1)^{p+q} x^{p} y^{q}\left(\begin{array}{c}
p+q \\
p
\end{array}\right) .
\end{aligned}
$$

Осталось вычислить степень дискриминанта, т.е. разность между коэффициентами при $X^{n-1} Y^{n-2}$ и $X^{n-2} Y^{n-1}$ в выражении для $F^{\prime}(a X+b Y)$. После некоторых преобразований получим

$$
\begin{aligned}
& \sum_{i=0}^{n-1}\left((-1)^{n} n(a-1)^{n-1-i} b^{i}\left(\begin{array}{c}
n-1 \\
i
\end{array}\right)+\left[\begin{array}{c}
n-1 \\
n-1-i
\end{array}\right]_{a-1, b}-\left[\begin{array}{c}
n-2 \\
n-1-i
\end{array}\right]_{a-1, b}\right) \\
& \times\left(\left[\begin{array}{c}
n-2 \\
i
\end{array}\right]_{a, b-1}-\left[\begin{array}{c}
n-2 \\
i-1
\end{array}\right]_{a, b-1}\right) \\
& \quad-\sum_{i=0}^{n-1}\left(\left[\begin{array}{c}
n-1 \\
n-1-i
\end{array}\right]_{a-1, b}-\left[\begin{array}{c}
n-2 \\
n-1-i
\end{array}\right]_{a-1, b}-1\right) \\
& \quad \times\left(\left\{\begin{array}{c}
n-2 \\
i
\end{array}\right\}_{a, b-1}-\left\{\begin{array}{c}
n-3 \\
i
\end{array}\right\}_{a, b-1}-\left\{\begin{array}{c}
n-2 \\
i-1
\end{array}\right\}_{a, b-1}+\left\{\begin{array}{c}
n-3 \\
i-1
\end{array}\right\}_{a, b-1}\right) .
\end{aligned}
$$

Это степень дискриминанта неприводимого $\mathrm{SL}_{n}$-модуля со старшим весом $(a-b) \varphi_{1}+b \varphi_{2}$.

Подставив $b=1$, получим

$$
\frac{\left(n^{2}-n\right) a^{n+1}-\left(n^{2}+n\right) a^{n-1}-2 n(-1)^{n}}{(a+1)^{2}} .
$$

В частности, при $a=2$ получим

$$
\frac{\left(3 n^{2}-5 n\right) 2^{n}-4 n(-1)^{n}}{18} .
$$

Теоремы 2 и $2^{\prime}$ доказаны.

\section{$\S 3 . \quad E$-регулярные алгебры}

ДокАЗАТЕЛЬСТво тЕОРЕмы 3. Пункт 2) доказывается аналогично п. 4) теоремы 1.

Покажем, что п. 1) следует из п. 3). Выберем в $V^{*}$ базис $\left\{f_{1}, \ldots, f_{n}\right\}$, двойственный к базису $\left\{e_{1}, \ldots, e_{n}\right\}$ в $V$. Пусть $U \in \operatorname{Gr}(n-1, V)$ - гиперплоскость $f_{1}=0$. Ясно, что $\widetilde{\mathscr{E}}=\mathrm{GL}_{n} \cdot M_{0}$, где $M_{0}=\widetilde{\mathscr{E}} \cap(U, P \mathscr{A})$. Далее, $M=Z \cap(U, P \mathscr{A})$ есть линейное подпространство всех алгебр, для которых $U$ является $(n-1)$-мерной подалгеброй. Пусть $P$ - параболическая подгруппа матрищ вида $\left(\begin{array}{cc}A & 0 \\ * & B\end{array}\right)$, 
$\mathfrak{u}$ - алгебра Ли матриц вида $\left(\begin{array}{cc}0 & X \\ 0 & 0\end{array}\right)$, где $B-((n-1) \times(n-1))$-матрица, $X-(1 \times(n-1))$-матрица. Всякая алгебра $A \in M$ определяет линейное отображение $\mathfrak{u} \rightarrow \Lambda^{n-1} U^{*} \otimes V / U$. Поскольку $A \in M_{0}$ тогда и только тогда, когда это отображение вырождено, $\operatorname{codim}_{M} M_{0}=1$. Легко видеть также, что $M_{0}$ неприводимо, поскольку $M_{0}$ является разнесением группой $P$ линейного подпространства

$$
M_{0}^{1}=\left\{A \in M_{0} \mid \text { алгебра } E_{12} A \text { имеет подалгебру } U\right\} .
$$

Отсюда следует, что $\widetilde{\mathscr{E}}$ является неприводимым дивизором в $Z$, и из п. 3 ) следует п. 1).

Докажем п. 3). Будем называть $(n-1)$-мерную подалгебру $U^{\prime}$ алгебры $A \in \mathscr{E}$ критической, если пара $\left(U^{\prime}, A\right)$ лежит в $\mathscr{\mathscr { E }}$. В этом случае найдется $(n-2)$-мерное подпространство $W^{\prime} \subset U^{\prime}$ такое, что если $V=U^{\prime} \oplus \mathbb{C} e$, $v \in \mathfrak{g l}_{n}-$ ненулевой линейный оператор такой, что $v(V) \subset \mathbb{C} e, v\left(W^{\prime}\right)=0$, то $U^{\prime}$ является подалгеброй алгебры $v A$ (см. доказательство п. 5) теоремы 1 ). Для доказательства п. 3 ) достаточно доказать, что в алгебрах из $M_{0}^{1}$ общего положения подалгебра $U$ является единственной критической подалгеброй. Пусть $N \subset M_{0}^{1}-$ подмногообразие всех алгебр, для которых сушествует другая критическая подалгебра.

Заметим, что $M_{0}^{1}$ нормализуется параболической подгруппой (9). Обозначим ее $Q$. Тогда $N$ является разносом группой $Q$ подмногообразий $N_{2}$ и $N_{3}$, где $A \in N_{i}$ тогда и только тогда, когда гиперплоскость $f_{i}=0$ является критической подалгеброй. В свою очередь $N_{2}$ является разносом векторных подпространств $N_{2}^{1}$ и $N_{2}^{3}$, а $N_{3}$ - разносом подпространств $N_{3}^{1}, N_{3}^{2}$ и $N_{3}^{4}$, где $N_{i}^{j} \subset N_{i}-$ подпространство тех алгебр, для которых $(n-2)$-мерное подпространство $W^{\prime}$ (см. выше) равно $f_{i}=f_{j}=0$. Обозначим $Q_{i}^{j} \subset Q$ подгруппу, нормализуюшую флаг $f_{i} \subset\left\langle f_{i}, f_{j}\right\rangle$. Тогда легкая проверка показывает, что

$$
\begin{gathered}
\operatorname{codim}_{Q} Q_{2}^{1}=1, \quad \operatorname{codim}_{Q} Q_{2}^{3}=n-1, \quad \operatorname{codim}_{Q} Q_{3}^{1}=n-1, \\
\operatorname{codim}_{Q} Q_{3}^{2}=n, \quad \operatorname{codim}_{Q} Q_{3}^{4}=2 n-3 .
\end{gathered}
$$

С другой стороны,

$$
\begin{gathered}
\operatorname{codim}_{M_{0}^{1}} N_{2}^{1}=n \\
\operatorname{codim}_{M_{0}^{1}} N_{2}^{3}=\operatorname{codim}_{M_{0}^{1}} N_{3}^{1}=\operatorname{codim}_{M_{0}^{1}} N_{3}^{2}=\operatorname{codim}_{M_{0}^{1}} N_{3}^{4}=2 n-2 .
\end{gathered}
$$

Отсюда во всех случаях $\operatorname{codim}_{M_{0}^{1}} Q N_{i}^{j} \geqslant 1$, и теорема доказана.

\section{§ 4. Регулярные 4-мерные антикоммутативные алгебры}

ДокаЗАТЕЛЬСТво теоремЫ 4. Докажем п. 1). Нужно доказать, что если $A$-4-мерная регулярная антикоммутативная алгебра со следом 0 , то ее трехмерные подалгебры $S_{1}, S_{2}, \ldots, S_{5}$ образуют конфигурацию гиперплоскостей обшего положения, т.е. пересечение любых трех одномерно, а любых четырех нульмерно. 
Действительно, пусть, скажем, $U=S_{1} \cap S_{2} \cap S_{3}$ двумерно. Пусть $v \in U, v \neq 0$. Тогда оператор $[v, \cdot]$ индуцирует линейный оператор в $A / U$, поскольку $U$ является подалгеброй. При этом $S_{1} / U, S_{2} / U$ и $S_{3} / U$ - его одномерные собственные подпространства. Поскольку $\operatorname{dim} A / U=2$, оператор является гомотетией. Так как это верно для любого $v \in U$, всякое трехмерное подпространство, содержащее $U$, является трехмерной подалгеброй, что противоречит тому, что их ровно пять.

Пусть теперь $U=S_{1} \cap S_{2} \cap S_{3} \cap S_{4}$ одномерно, $v \in U, v \neq 0 . \quad$ Тогда оператор $[v, \cdot]$ индуцирует оператор в $A / U$. У этого оператора есть четыре собственных двумерных подпространства $S_{1} / U, \ldots, S_{4} / U$ таких, что любые три из них имеют нулевое пересечение (см. выше). Отсюда следует, что этот оператор является гомотетией. Пусть $W \supset U$ - произвольное двумерное подпространство, $w \in W$ - вектор, не пропорциональный $v$. Тогда оператор $[w, \cdot]$ индуцирует линейньй оператор в $A / W$. Пусть $z$ - его ненулевой собственный вектор. Тогда $\langle v, w, z\rangle$ - трехмерная подалгебра. Значит, каждый вектор включается в трехмерную подалгебру, что противоречит тому, что их ровно пять.

Это рассуждение также показывает, что в $A$ нет одномерных идеалов. Поскольку всякое трехмерное подпространство, содержашее двумерный идеал, является подалгеброй, двумерных идеалов также нет. Это доказывает п. 2).

Докажем п. 3). Пусть $X$ - многообразие двумерных подалгебр в $A$; тогда $X \subset \operatorname{Gr}(2,4)$. Рассмотрим также вложение Плюккера $\operatorname{Gr}(2,4) \subset \mathbb{P}^{5}=P\left(\Lambda^{2} \mathbb{C}^{4}\right)$. Проверим сначала, что вложение $X \subset \mathbb{P}^{5}$ не вырождено, т.е. образ не содержится ни в какой гиперплоскости. Пусть $S_{1}, S_{2}, S_{3}, S_{4}$ - какие-то четыре трехмерных подалгебры. Поскольку они образуют конфигурацию общего положения, можно выбрать такой базис $\left\{e_{1}, e_{2}, e_{3}, e_{4}\right\}$ в $A$, что $S_{i}=\left\langle e_{1}, \ldots, \hat{e}_{i}, \ldots, e_{4}\right\rangle$. Поскольку пересечение трехмерных подалгебр есть двумерная подалгебра, в $A$ есть шесть подалгебр $\left\langle e_{i}, e_{j}\right\rangle, i \neq j$. Соответствуюшие бивекторы $e_{i} \wedge e_{j}$ образуют базис в $\Lambda^{2} \mathbb{C}^{4}$, поэтому не могут лежать ни в какой гиперплоскости.

Итак, $H^{0}\left(X, \mathscr{O}_{X}(1)\right)=\Lambda^{2} \mathbb{C}^{4}$. Чтобы доказать, что $X$ является поверхностью дель Пещь степени пять, осталось проверить, что $\mathscr{O}_{X}(1)$ совпадает с антиканоническим пучком (см. [3]). Поскольку $Y=\operatorname{Gr}(2,4)$ является квадрикой в $\mathbb{P}^{5}$, имеем $\omega_{Y}=\mathscr{O}_{Y}(2-5-1)=\mathscr{O}_{Y}(-4)$. Множество $X$ является неособым подмногообразием в $Y$ коразмерности 2 , поэтому $\omega_{X}=\omega_{Y} \otimes \Lambda^{2} \mathscr{N}_{X / Y}$, где $\mathscr{N}_{X / Y}-$ нормальный пучок. Далее, $X$ является схемой нулей регулярного сечения расслоения $\mathscr{L}=\Lambda^{2} \mathscr{S}^{*} \otimes V / \mathscr{S}$ (см. доказательство теоремы 1$)$, откуда $\mathscr{N}_{X / Y}=\left.\mathscr{L}\right|_{Y}$ и $\Lambda^{2} \mathscr{N}_{X / Y}=\mathscr{O}_{X}(3)$, поскольку $c_{1}(\mathscr{L})=3 H$. Окончательно получаем

$$
\omega_{X}=\mathscr{O}_{X}(-4) \otimes \mathscr{O}_{X}(3)=\mathscr{O}_{X}(-1),
$$

что и требовалось доказать.

Осталось доказать п. 4). Заметим, что поскольку $X$ является поверхностью дель Пецщо степени пять, на ней лежит десять прямых, а поскольку вложение $X \subset P\left(\Lambda^{2} \mathbb{C}^{4}\right)$ является антиканоническим, эти прямые являются обычными прямыми в $P\left(\Lambda^{2} \mathbb{C}^{4}\right)$, лежащими на $\operatorname{Gr}(2,4)$. Осталось установить биекцию между этими прямыми и веерами. Если $b \in \Lambda^{2} \mathbb{C}^{4}$, то $b$ принадлежит конусу над $\operatorname{Gr}(2,4)$ тогда и только тогда, когда $b \wedge b=0$. Поэтому если $b_{1}, b_{2}$ принадлежат этому конусу, то прямая, их соединяюшая, принадлежит конусу тогда и только тогда, когда $b_{1} \wedge b_{2}=0$. Но это в точности - условие веера. 


\section{Список литературы}

1. Бернштейн И. Н., Гельфанд И. М., Гельфанд С. И. Клетки Шуберта и когомологии пространств $G / P / /$ Укр. матем. ж. 1973. Т. 28. №3. С. 3-26.

2. Клейман С. Л. Численная теория особенностей // Укр. матем. ж. 1980. Т. 35. №6. C. $69-148$.

3. Манин Ю. И. Кубические формы. М.: Наука, 1972.

4. Мамфорд Д. Комплексные проективные многообразия. М.: Мир, 1979.

5. Фултон У. Теория пересечения. М.: Мир, 1989.

6. Bergman G. M. The diamond lemma for ring theory // Adv. in Math. 1978. V. 29. № 2. P. $178-218$.

7. Bott R. Homogeneous vector bundles // Ann. of Math. 1957. V. 66. № 2. P. 203-248.

8. De Concini C., Weyman J. A formula with nonnegative terms for the degree of the dual variety of a homogeneous space // Proc. Amer. Math. Soc. 1997. V. 125. № 1. P. 1-8.

9. Knop F., Menzel G. Duale Varietäten von Fahnenvarietäten // Comment. Math. Helv. 1987. V. 62. № 1. P. 38-61.

10. Lascoux A. Degree of the dual Grassman variety // Comm. Algebra. 1981. V. 9. № 11 . P. $1215-1225$.

11. Tevelev E. A. Generic Algebras // Transformation Groups. 1996. V. 1. № 1-2. P. 127-151.

Московский государственный университет

Поступило в редакцию им. М. В. Ломоносова 